\title{
Optimal Transmit Power in Wireless Sensor Networks
}

\author{
Sooksan Panichpapiboon, Student Member, IEEE, Gianluigi Ferrari, Member, IEEE, and \\ Ozan K. Tonguz, Member, IEEE
}

\begin{abstract}
Power conservation is one of the most important issues in wireless ad hoc and sensor networks, where nodes are likely to rely on limited battery power. Transmitting at unnecessarily high power not only reduces the lifetime of the nodes and the network, but also introduces excessive interference. It is in the network designer's best interest to have each node transmit at the lowest possible power while preserving network connectivity. In this paper, we investigate the optimal common transmit power, defined as the minimum transmit power used by all nodes necessary to guarantee network connectivity. This is desirable in sensor networks where nodes are relatively simple and it is difficult to modify the transmit power after deployment. The optimal transmit power derived in this paper is subject to the specific routing and medium access control (MAC) protocols considered; however, the approach can be extended to other routing and MAC protocols as well. In deriving the optimal transmit power, we distinguish ourselves from a conventional graph-theoretic approach by taking realistic physical layer characteristics into consideration. In fact, connectivity in this paper is defined in terms of a quality of service (QoS) constraint given by the maximum tolerable bit error rate (BER) at the end of a multihop route with an average number of hops.
\end{abstract}

Index Terms—Ad hoc wireless networks, sensor networks, power control, connectivity.

\section{INTRODUCTION}

T $\mathrm{N}$ an ad hoc wireless network, where nodes are likely to Loperate on limited battery life, power conservation is an important issue. Conserving power prolongs the lifetime of a node and also the lifetime of the network as a whole. In addition, transmitting at low power reduces the amount of excessive interference. The fundamental question which naturally arises is: "What is the optimal transmit power to be used?" This is the fundamental question that we try to answer in this paper. Obviously, a suitable criterion of optimality has to be introduced.

One of the goals of forming a network is to have network connectivity - that is, each node should be able to communicate with any of the other nodes, possibly via multiple hops. The connectivity level of an ad hoc wireless network depends on the transmit power of the nodes. If the transmit power is too small, the network might be disconnected (i.e., there may be multiple disconnected clusters of nodes instead of a single overall connected network). However, as mentioned earlier, transmitting at excessively high power is inefficient because of the mutual interference in the shared radio channel and the limited battery lifetime. Thus, it is intuitively clear that the optimal transmit power is the minimum power sufficient to guarantee network connectivity [1], [2], [3].

- S. Panichpapiboon and O.K. Tonguz are with the Electrical and Computer Engineering Department, Carnegie Mellon University, 5000 Forbes Ave., Pittsburgh, PA 15213-3890.

E-mail:sooksan@cmu.edu, tonguz@ece.cmu.edu.

- G. Ferrari is with the Dipartimento di Ingegneria dell'Informazione, University of Parma, Viale G.P. Usberti, 181/A, I-43100 Parma, Italy. E-mail: gianluigi.ferrari@unipr.it.

Manuscript received 11 Feb. 2005; revised 22 Aug 2005; accepted 5 Nov. 2005; published online 16 Aug. 2006.

For information on obtaining reprints of this article, please send e-mail to: tmc@computer.org, and reference IEEECS Log Number TMC-0029-0205.
Ideally, the transmit power of a node should be adjusted on a link-by-link basis to achieve the maximum possible power savings [2], [3], [4], [5], [6]. Nonetheless, due to the absence of a central controller in a "pure" ad hoc network with flat architecture, performing power control on a linkby-link basis is a complicated and cumbersome task. A simpler solution, which is more viable for implementation, is to have all the nodes use a common transmit power. This is desirable in sensor networks where nodes are relatively simple and it is difficult to modify the transmit power after deployment. In addition, the performance difference, in terms of traffic carrying capacity, between adjusting the power locally and employing a common transmit power is small, especially when the number of nodes is large [1].

In this paper, we investigate the optimal transmit power for an ad hoc wireless networking scenario where all nodes use a common transmit power. Although the optimal common transmit power derived in this paper is subject to the routing and the medium access control (MAC) protocol considered, the approach can be extended to other routing and MAC protocols as well. Other studies which consider common transmit power exist [1], [7], [8], [9]. In [7] and [8], the minimal transmission range at which a network is connected with high probability is studied. In [9], the authors investigate the minimal common transmit power sufficient to preserve network connectivity. These works, however, follow a graph-theoretic approach which only takes into account the distances between nodes. More specifically, the authors consider that two neighboring nodes can communicate if they are within the communication range of each other and two nodes that are not neighbors can communicate if there is a multihop path connecting them. It is important to point out that, although there may be a path connecting two nodes, communication 
between them may not be possible as the quality of service (QoS) in terms of tolerable bit error rate (BER) at the end of a multihop route may not be satisfied. We discuss this in more detail in Section 3. As opposed to the conventional graph-theoretic approach, in this paper, the optimal transmit power sufficient to maintain network connectivity is found according to a physical layer-oriented QoS constraint given by the maximum tolerable BER at the end of a multihop route with an average number of hops.

In this paper, we evaluate the optimal transmit power both analytically (in the case of regular topology) and via simulations (in the case of random topology). Moreover, we investigate the interrelation between optimal transmit power, data rate, and node spatial density. In addition to our earlier work [10], this paper also investigates 1) the impact of different propagation pathloss exponents (on different links of a multihop route) on the performance of a common transmit power control scheme and 2) the interrelation between transmit power, connectivity, and network longevity. Furthermore, in this paper, we provide a rigorous analysis of exact interference power and BER using a detection theory approach, as opposed to simply assuming that the interference on each link is the average power as considered in [10]. We also validate our analysis with simulation.

The rest of this paper is organized as follows: In Section 2, we describe the model and the assumptions that will be used in the derivation of the optimal transmit power. In Section 3, we define network connectivity. We introduce a communication-theoretic model for evaluating route BER in Section 4 . The minimum transmit power sufficient to maintain network connectivity in both regular and random topologies is analyzed in Section 5. The performance in terms of node/network lifetime and effective transport capacity is evaluated in Section 6. Numerical results, along with their implications, are presented in Section 7. Finally, we provide a discussion of related work and conclusions in Section 8 and Section 9, respectively.

\section{Model AND Assumptions}

In this section, we describe the basic ad hoc wireless network communication model and the basic assumptions considered in the this paper.

\subsection{Network Topology}

Throughout the paper, we consider a scenario where $N$ nodes are distributed over a surface with finite area $A$. The node spatial density is defined as the number of nodes per unit area and is denoted as $\rho_{\mathrm{s}} \triangleq N / A$. To avoid edge effects, we assume the network surface to be the surface of a torus with length $2 R$ on each edge, as shown in Fig. 1. However, the analytical technique presented in this paper can be applied to other types of surfaces as well. In a real scenario, the performance predicted by our analysis may not be extremely precise for nodes on the edge of the network surface. In this case, a more precise performance evaluation may be obtained via simulations. Nonetheless, the results presented in this paper provide a representative description of a realistic network behavior.

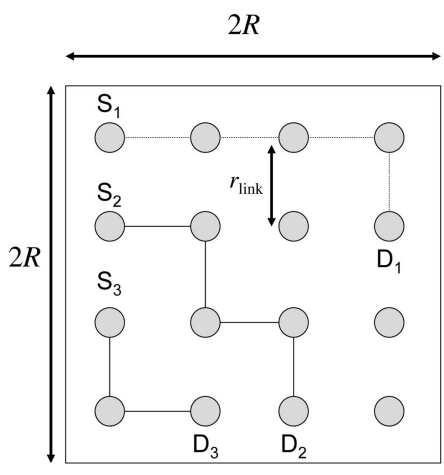

(a)

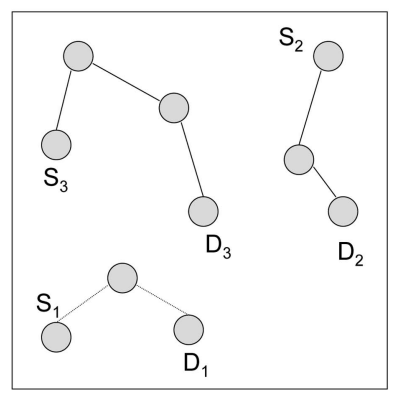

(b)
Fig. 1. Possible topologies: (a) regular and (b) random. In each case, examples of multihop routes are shown.

In addition to a simple scenario with square grid network topology considered in [10], in this paper, we also consider a realistic scenario with two-dimensional Poisson node distribution. In a network with square grid topology, shown in Fig. 1a, each node has four nearest neighbors at a fixed distance. In contrast, the positions of nodes in a network with a two-dimensional Poisson topology are random and independent of each other, as shown in Fig. 1b. The probability mass function (pmf) of the number of nodes $N_{a}$ over a surface of area $a$ in the case with two-dimensional Poisson topology is given by

$$
\operatorname{Pr}\left(N_{a}=j\right)=\frac{\left(\rho_{\mathrm{s}} a\right)^{j}}{j !} e^{-\rho_{\mathrm{s}} a} \quad j=0,1,2, \ldots,
$$

where, in this case, $\rho_{\mathrm{s}}$ corresponds to the average number of nodes per unit area or the average node spatial density. Considering the same value of $\rho_{\mathrm{s}}$ for both types of networks with regular and random topologies makes the comparison between them fair and meaningful.

In this paper, we only consider ad hoc wireless networks with stationary nodes. Examples of such networks are sensor networks [11] and wireless mesh networks [12]. The extension to a scenario where nodes are mobile can be done following the approach proposed in [13].

\subsection{Routing}

We assume a simple routing strategy such that a packet is relayed hop-by-hop, through a sequence of nearest neighboring nodes, until it reaches the destination. In addition, we assume that a source node discovers a route prior to data transmission [14]. Discovery of a multihop route from a source to a destination is a crucial phase in a wireless networking scenario with flat architecture. The focus of this paper, however, is on the characterization of the steady state behavior of on-going peer-to-peer multihop communications. Therefore, we will assume that a route between source and destination exists. We discuss routing in networks with regular and random topologies in the following sections.

\subsubsection{Square Grid Topology}

Due to the regularity of this topology, the distance to the nearest neighbor, denoted by $r_{\text {link }}$, is fixed, and a route is 


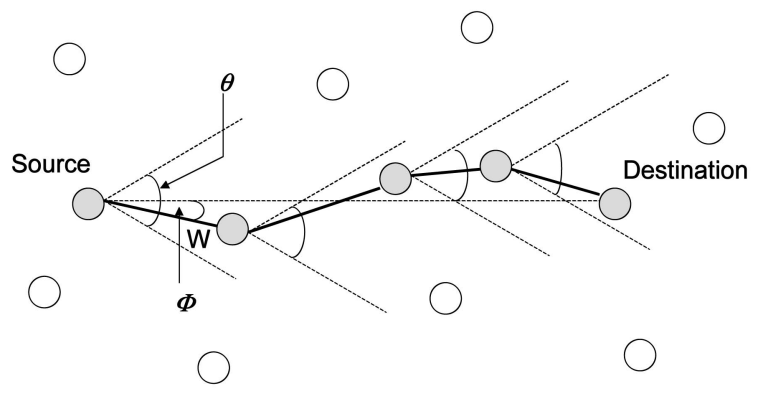

Fig. 2. Possible multihop route in a random topology. $W$ corresponds to the distance to the nearest neighbor, $\Phi$ corresponds the projection angle, and $\theta$ corresponds to the angle which a node looks for a neighbor in the direction of the destination.

constituted by a sequence of hops with equal length. The distance $r_{\text {link }}$ can be computed as follows: One can first observe that constructing a square lattice of $N$ nodes over a surface of a torus with area $A$ is equivalent to fitting $N$ small square tiles of area $r_{\text {link }}^{2}$ into a large square of area $A$. Hence, it must hold that $N r_{\text {link }}^{2}=A$ and, therefore, the distance to the nearest neighbor can be written as

$$
r_{\text {link }}=\sqrt{\frac{A}{N}}=\frac{1}{\sqrt{\rho_{\mathrm{s}}}} .
$$

\subsubsection{Two-Dimensional Poisson Topology}

In the case of random topology, we still consider a routing scheme where each intermediate node in a multihop route relays the packets to its nearest neighbor in the direction of the destination. In particular, we assume that an intermediate node in the route selects the nearest node within a sector of angle $\theta$ toward the direction of the destination as the next hop [15]. An example of a multihop route constructed in this way is shown in Fig. 2. In this case, a route can be visualized as a deviation from the straight line between source and destination, referred to as reference path. Unlike in a scenario with grid topology, in a network with two-dimensional Poisson topology, the distance from a node to its nearest neighbor is not a constant. Let $W$ be a random variable denoting the distance to the nearest neighbor in a twodimensional Poisson node distribution. It can be shown that, keeping the node spatial density fixed, for large $N$ (i.e., as $N \rightarrow \infty$ ), the CDF of the distance to the nearest neighbor in a torus is

$$
F_{W}(w)= \begin{cases}0 & w<0 \\ 1-e^{-\rho_{\mathrm{s}} \pi w^{2}} & 0 \leq w<R \\ 1-e^{-\rho_{\mathrm{s}} \chi} & R \leq w<\sqrt{2} R \\ 1 & w \geq \sqrt{2} R\end{cases}
$$

where $\chi \triangleq\left[4 w^{2}\left(\frac{\pi}{4}-\xi\right)+4 R \sqrt{w^{2}-R^{2}}\right]$ and $\xi \triangleq \cos ^{-1}\left(\frac{R}{w}\right)$. Note that the total surface area of a torus with length $2 R$ on each edge is equal to $4 R^{2}$. Hence, the node spatial density in this case is $\rho_{\mathrm{s}}=N / 4 R^{2}$. Substituting this value back in (3) and computing $F_{W}(R)$, it follows that $\operatorname{Pr}[W \leq R]$, i.e., $F_{W}(R)$, is almost 1 if $N \geq 10$. In other words, $W$ is almost surely lower than $R$. Consequently, for $N \gg 10$ (which will be true for all scenarios considered in this paper), we can neglect the case where $W>R$ without significant impact on the accuracy of the analytical results.

Using the fact that $W \leq R$ with very high probability and generalizing the $\mathrm{CDF}$ given in (3) to a scenario where a node looks for a neighboring node within a sector of angle $\theta$, the CDF of $W$ can be written as

$$
F_{W}^{(\theta)}(w)= \begin{cases}1 & w>R \\ 1-e^{-\rho_{s} \frac{\theta w^{2}}{2}} & 0 \leq w \leq R \\ 0 & \text { otherwise. }\end{cases}
$$

More details on the derivation can be found in [16].

\subsection{Medium Access Control Protocol}

In this paper, we consider a simple reservation-based MAC protocol introduced in [17] and defined as reserve-and-go (RESGO). ${ }^{1}$ In this protocol, a source node first reserves intermediate nodes on a route for relaying its packets to the destination-characterization of this phase is beyond the scope of this paper. A transmission can begin after a route is discovered and reserved. The main idea of the protocol is that a source node or a relay node generates an exponential random backoff time before it transmits or relays each packet. After the random backoff time expires, a node can start transmitting a packet. The random backoff time helps reduce interference among nodes in the same route and also among nodes in different routes. Throughout this paper, we assume that the random backoff time is exponential with mean $1 / \lambda_{t}$. In other words, given that a node has packets to send, packets are transmitted with rate $\lambda_{\mathrm{t}}$ (dimension: $[\mathrm{pck} / \mathrm{s}]$ ). Note that this is generally different from the traffic generation rate, $\lambda_{\mathrm{g}}$.

\section{Connectivity}

As discussed earlier, the optimal common transmit power is the minimum power sufficient to preserve network connectivity. In this section, we formalize the definition of network connectivity. Conceptually, an ad hoc wireless network is often viewed as a graph, where vertices represent the nodes and edges represent the links connecting neighboring nodes. From a graph-theoretical perspective, a network is connected if there is a path (possibly multihop) connecting any node to any other node in the network. However, using this notion of connectivity for an ad hoc wireless network, where a communication channel is error-prone, can be misleading. Since the wireless links are susceptible to errors, the QoS in terms of route BER deteriorates as the number of hops in a route increases. Consequently, the performance may be unacceptable, although there is a sequence of links to the destination.

In order to take the physical layer characteristics into account, in this paper, we consider network connectivity from a communication-theoretic viewpoint. In particular, a network is said to be connected if any source node can communicate with a BER lower than a prescribed value $\mathrm{BER}_{\mathrm{th}}$ to a destination node placed at the end of a multihop

1. This MAC protocol was erroneously referred to in [17] as the Aloha MAC protocol for its resemblance to the classical Aloha MAC protocol [18]. However, there are significant differences which make the proposed protocol different from the classical Aloha MAC protocol: 1) multihop route reservation and 2) no use of retransmission techniques. 


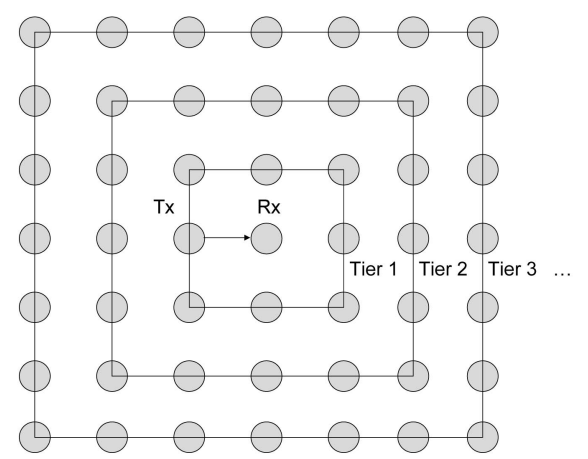

Fig. 3. Tier structure of a network with square grid topology.

route with an average number of hops [19]. To be conservative, in most of this paper, we consider an ideal worst-case scenario where an information bit is relayed on each link of a route toward a destination without retransmissions. However, it will be shown that use of retransmission techniques lowers the BER and thus decreases the minimum power required to maintain the network connectivity. We will discuss this in more detail in Section 7.1.

In addition, note that this notion of connectivity corresponds to requiring that, on average, a communication between a source and a destination can be guaranteed with a desired quality. However, it does not guarantee that a source can communicate with every node in the network with this QoS. A more stringent connectivity requirement, such that a source can communicate with every node in the network with the desired QoS, can also be enforced. The approach proposed in this paper can be straightforwardly extended by considering the BER at the end of a multihop route with the maximum possible number of hops. In the next section, we derive a simple analytical expression for the average number of hops in the cases with grid and random topologies.

\subsection{Square Grid Topology}

Due to the spatial invariance on a torus, we can assume without any loss of generality that a source node is at the center of the network (see Fig. 3). If a destination node is selected at random, the minimum number of hops to reach the destination can range from 1 to $2 i_{\max }$, where $i_{\max }$ is the maximum tier order. In other words, it takes 1 hop to reach a destination which is a neighbor of a source node in Tier 1, and it takes $2 i_{\max }$ hops to reach the farthest node from the center in Tier $i_{\max }$. The average number of hops can be obtained by counting the number of hops on a route from the source to each destination node and finding the average value. Assuming that each destination is equally likely, the average number of hops on a route can be written as

$$
\bar{n}_{\text {grid }}=\frac{1}{N-1}\left[4 \sum_{i=1}^{i_{\max }} i+4 \sum_{i=1}^{i_{\max }} 2 i+8 \sum_{i=1}^{i_{\max }} \sum_{j=1}^{i-1}(i+j)\right] .
$$

The first summation term in (5) corresponds to the number of hops it takes to reach any of the four nodes in alignment with the source at the center of the network in all possible tiers, the second summation corresponds to the number of hops to reach nodes on the four corners of each tier, and, finally, the third summation corresponds to the number of hops to reach the other nodes in each tier. With straightforward algebra, (5) can be simplified to

$$
\bar{n}_{\text {grid }}=\frac{2}{N-1}\left(2 i_{\max }^{3}+3 i_{\max }^{2}+i_{\max }\right) .
$$

Since $i_{\max } \simeq \sqrt{N} / 2$, when the number of nodes is sufficiently large, from (6), one obtains:

$$
\bar{n}_{\text {grid }} \simeq \frac{\sqrt{N}}{2}+\frac{1}{\sqrt{N}}+\frac{3}{2} \simeq \frac{\sqrt{N}}{2} .
$$

\subsection{Two-Dimensional Poisson Topology}

In this case, we define an average route as a route between a source and a destination separated by an average Euclidean distance between two randomly chosen points on a torus. We now propose an approach for computing the average path length. Let $Z$ be the random variable denoting the distance between a source and a destination. It can be shown that the pdf of $Z$ is [15]

$$
f_{Z}(z)= \begin{cases}\frac{\pi z}{2 R^{2}} & 0 \leq z<R \\ \frac{\pi z}{2 R^{2}}-\frac{2 z \cos ^{-1}(R / z)}{R^{2}} & R \leq z<\sqrt{2} R .\end{cases}
$$

Based on (8), the following average distance between a source and a destination node can be obtained:

$$
\bar{Z}=\int_{0}^{\sqrt{2} R} z f_{Z}(z) \mathrm{d} z=\frac{R}{3}[\sqrt{2}+\ln (1+\sqrt{2})] .
$$

Intuitively, the average number of hops in a route must be proportional to the average hop length, i.e., the average distance between two neighbors. For instance, one can expect that a route will consist of many hops if each hop length is short. In our analysis, we assume that each hop deviates, with respect to the reference path, by an angle $\Phi$, where $\Phi$ is uniformly distributed in the interval $(-\theta / 2, \theta / 2)$ - the angles $\Phi$ and $\theta$ are shown in the example considered in Fig. 2. By projecting each hop onto the reference path, the average number of hops between a source and a destination can be approximated as follows: ${ }^{2}$

$$
\bar{n}_{\text {rand }} \simeq \frac{\bar{Z}}{\mathrm{E}[W \cos \Phi]},
$$

where $\mathrm{E}[W \cos \Phi]$ is the average projected hop length. In general, $W$ and $\Phi$ are not independent. However, as discussed in Section 2.1, since it is highly likely that $W \leq R$, in this case, it can be shown that $W$ and $\Phi$ are basically independent. Therefore, it follows that

$$
\mathrm{E}[W \cos \Phi] \simeq \mathrm{E}[W] \mathrm{E}[\cos \Phi] .
$$

The expected values of $W$ and $\cos \Phi$ can be straightforwardly obtained from the pdfs of $W$ and $\Phi$. In fact, assuming

2. To be precise, in the derivation of $\bar{n}_{\text {rand }}$ in (10), one should consider $\mathrm{E}[W \cos \Phi / \theta]$ at the denominator on the right-hand side. However, in the remainder of this paper, we assume that $\theta$ is given and all the expected values have to be interpreted as conditional expectations. In general, the angle $\theta$ is likely to be known. For example, in a routing protocol where a node does not select the next relay node that is in the opposite direction of the destination, the angle $\theta$ is $\pi$. 
that the network area is large (i.e., $R$ is large), it can be shown that $\mathrm{E}[W] \simeq \sqrt{\pi /\left(2 \rho_{\mathrm{s}} \theta\right)}$ and $\mathrm{E}[\cos \Phi] \simeq(2 / \theta) \sin (\theta / 2)$ [15]. Finally, using (9), (10), and the simplified expression for $\mathrm{E}[W \cos \Phi]$, it can be shown that

$$
\bar{n}_{\text {rand }} \simeq \frac{\sqrt{N} \sqrt{\theta^{3}}[\sqrt{2}+\ln (1+\sqrt{2})]}{6 \sqrt{2 \pi} \sin \left(\frac{\theta}{2}\right)},
$$

where we have used the fact that $\rho_{\mathrm{s}}=N / A$.

\section{Ber at the End of a Multihop Route}

Since the network connectivity is defined in terms of BER quality at the end of a multihop route, in this section, we present an approach to evaluate the route BER.

\subsection{Square Grid Topology}

\subsubsection{BER with Exact Interference Analysis}

In this section, we analyze the link BER and the route BER using a detection-theoretic approach. Generally, the received signal observed at the receiver is the sum of three components: 1) the intended signal from a transmitter, 2) the interfering signals from other nodes, and 3) the thermal noise. Since the interfering signals come from other nodes, we assume that the total interfering signal can be treated as an additive noise process independent of the thermal noise process. The received signal $r$ during each bit period can be expressed as

$$
r=s_{\text {sig }}+\sum_{j=1}^{N-2} s_{j}+w_{\text {thermal }},
$$

where $s_{\text {sig }}$ is the signal from the transmitter, $s_{j}$ is the signal from an interfering node $j$, and $w_{\text {thermal }}$ is the thermal noise signal. In the following, we will derive these three components.

Consider a link between the transmitter and the receiver shown in Fig. 3. Assuming that a signal is attenuated with a distance raised to the power $\gamma$, where $\gamma$ is the pathloss exponent, the power of the intended signal from the transmitter as observed at the receiver can be written as [20]

$$
P_{\mathrm{r}}=\frac{\alpha P_{\mathrm{t}}}{r_{\text {link }}^{\gamma}},
$$

where

$$
\alpha \triangleq \frac{G_{\mathrm{t}} G_{\mathrm{r}} c^{2}}{(4 \pi)^{2} f_{\mathrm{c}}^{2}}
$$

and $P_{\mathrm{t}}$ is the transmit power, $G_{\mathrm{t}}$ and $G_{\mathrm{r}}$ are the transmitter and receiver antenna gains, $f_{\mathrm{c}}$ is the carrier frequency, and $c$ is the speed of light. In this paper, we assume that the antennas at the nodes are omnidirectional $\left(G_{\mathrm{t}}=G_{\mathrm{r}}=1\right)$, and the carrier frequency is in the unlicensed $2.4 \mathrm{GHz}$ band. Assuming a binary phase shift keying (BPSK) modulation, there can be two cases for the amplitude of the received signal: 1) $s_{\mathrm{sig}}=\sqrt{P_{\mathrm{r}} / R_{\mathrm{b}}} \triangleq \sqrt{E_{\mathrm{b}}}$ if a " +1 " is transmitted and 2) $s_{\text {sig }}=-\sqrt{P_{\mathrm{r}} / R_{\mathrm{b}}} \triangleq-\sqrt{E_{\mathrm{b}}}$ if a " -1 " is transmitted-note that $E_{\mathrm{b}}$ is the bit energy of the received signal.
The thermal noise power can be written as follows:

$$
P_{\text {thermal }}=F k T_{0} B \text {, }
$$

where $F$ is the noise figure, $k=1.38 \times 10^{-23} \mathrm{~J} / \mathrm{K}$ is the Boltzmann's constant, $T_{0}$ is the room temperature $\left(T_{0}=300 \mathrm{~K}\right)$, and $B$ is the transmission bandwidth. The received thermal noise signal is simply $w_{\text {thermal }}=\sqrt{F k T_{0} B}$.

Due to spatial invariance (torus assumption), without any loss of generality, one could compute the amount of interference experienced by a receiving node as if it were at the center of the network. Fig. 3 illustrates a scenario where the receiver is at the center of the network and the other nodes are grouped in concentric square tiers. Consider a potential interfering node $j$ at a distance $\nu_{j} r_{\text {link }}$ from the receiver, where $\nu_{j}$ is a multiplicative factor which depends on the position of node $j$. For example, a node at the corner of the first tier would have a multiplicative factor $\nu=\sqrt{2}$ because it is at the distance $\sqrt{2} r_{\text {link }}$ from the receiver. The interference power from node $j$ can be written as

$$
P_{\text {int } j}=\frac{\alpha P_{\mathrm{t}}}{\left(\nu_{j} r_{\text {link }}\right)^{\gamma}}=\frac{P_{\mathrm{r}}}{\nu_{j}^{\gamma}} .
$$

For each interfering node $j$, the amplitude of the interfering signal can be classified into one of these three cases: 1) $s_{j}=\sqrt{P_{\mathrm{int} j} / R_{\mathrm{b}}}=\sqrt{E_{\mathrm{b}} / \nu_{j}^{\gamma}}$ if a " $+1^{\prime \prime}$ is transmitted, 2) $s_{j}=-\sqrt{P_{\mathrm{int} j} / R_{\mathrm{b}}}=-\sqrt{E_{\mathrm{b}} / \nu_{j}^{\gamma}}$ if a " -1 " is transmitted, and 3) $s_{j}=0$ if node $j$ does not transmit. The probability that an interfering node will transmit and cause interference depends on the MAC protocol employed. Considering the RESGO MAC protocol and assuming that each node transmits packets with fixed length $L$ (dimension: [b/pck]), it can be shown that the interference probability is equal to the probability that an interfering node transmits during a vulnerable interval of duration $L / R_{\mathrm{b}}$ [17]. This probability can be written as ${ }^{3}$

$$
p_{\text {tran }}=1-e^{-\frac{\lambda_{\mathrm{t}} L}{R_{\mathrm{b}}}} \text {. }
$$

The link BER can now be computed. Since we are considering a linear binary modulation, without loss of generality, we can assume that the transmitter transmits " +1 ". Thus, in this case, $s_{\text {sig }}=\sqrt{E_{\mathrm{b}}}$. Let us define a random vector $\vec{S}_{\text {int }}=\left\{s_{1}, s_{2}, \ldots, s_{N-2}\right\}$, where $s_{j}$ is the amplitude of the signal of an interfering node $j$ received at the receiver. Note that $\vec{S}_{\text {int }}$ is random because each $s_{j}$ can take one of these three different values with the probability given below:

3. Actually, this is the probability that a node will transmit given that it always has a packet to send. This is a pessimistic assumption, as there could be occasions when a node has nothing to send. Thus, the actual probability that a node will transmit should be lower. After a careful analysis, we have found that the actual probability that a relay node will transmit does not have a simple closed-form expression. This is partly due to the fact that the arrival process of the relay node is no longer a Poisson process. Thus, we believe that it is better to use a pessimistic assumption which allows us to derive an expression for a transmission probability that provides insight into the problem. Since we are using a pessimistic assumption, the actual performance with the RESGO MAC protocol is expected to be better than what is predicted by our analysis. 


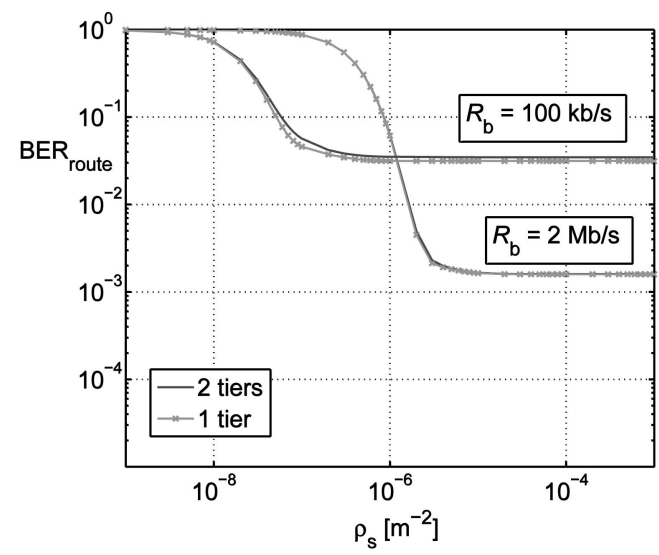

Fig. 4. Route BER as a function of node spatial density, comparing the case where only the interference from the first tier is considered and the case where the interference from the first two tiers is considered.

$$
s_{j}= \begin{cases}\sqrt{E_{\mathrm{b}} / \nu_{j}^{\gamma}} & \text { with probability } \frac{1}{2} p_{\operatorname{tran}} \\ -\sqrt{E_{\mathrm{b}} / \nu_{j}^{\gamma}} & \text { with probability } \frac{1}{2} p_{\operatorname{tran}} \\ 0 & \text { with probability } 1-p_{\operatorname{tran}} .\end{cases}
$$

Assuming that the threshold for bit detection is placed at 0 , the bit error probability can be written as

$$
\begin{aligned}
& P\{\text { bit error }\}=\mathrm{BER}_{\text {link }}= \\
& \sum_{\vec{S}_{\text {int }}} P\left\{\sqrt{E_{\mathrm{b}}}+\sum_{j=1}^{N-2} s_{j}+w_{\text {thermal }}<0 \mid \vec{S}_{\text {int }}\right\} P\left\{\vec{S}_{\text {int }}\right\}
\end{aligned}
$$

where the summation is carried out over all possible interference configurations given by $\vec{S}_{\text {int }}$. Given the random vector $\vec{S}_{\text {int }}$, the conditional error probability can be expressed as

$$
\begin{aligned}
& P\left\{\sqrt{E_{\mathrm{b}}}+\sum_{j=1}^{N-2} s_{j}+w_{\text {thermal }}<0 \mid \vec{S}_{\mathrm{int}}\right\} \\
& =Q\left(\frac{\sqrt{E_{\mathrm{b}}}+\sum_{j=1}^{N-2} s_{j}}{\sigma}\right)
\end{aligned}
$$

where $\sigma=\sqrt{F k T_{0} / 2}$. Assuming that a bit detected erroneously at the end of a link is not corrected in successive links, the BER at the end of a route with $\bar{n}_{\text {grid }}$ links, denoted as $\mathrm{BER}_{\text {route, }}^{\text {grid }}$ can be written as

$$
\mathrm{BER}_{\text {route }}^{\text {grid }}=1-\left(1-\mathrm{BER}_{\text {link }}\right)^{\bar{n}_{\text {grid }}} .
$$

Since we assume that each intermediate node decodes the signal before forwarding to the next node in a route, this is regarded as decode-and-forward transmission. The case where each intermediate node simply combines the received signal and relay the signal without decoding can also be considered following the approach in [21], [22].

Note that the size of the vector $\vec{S}_{\text {int }}$ increases as the number of nodes in the network increases. Moreover, the values that $\vec{S}_{\text {int }}$ can take (and, hence, its pmf) are of the order of $3^{N}$. Thus, considering all nodes in the network as potential sources of interference may not be practical. However, our study shows that the dominant interference

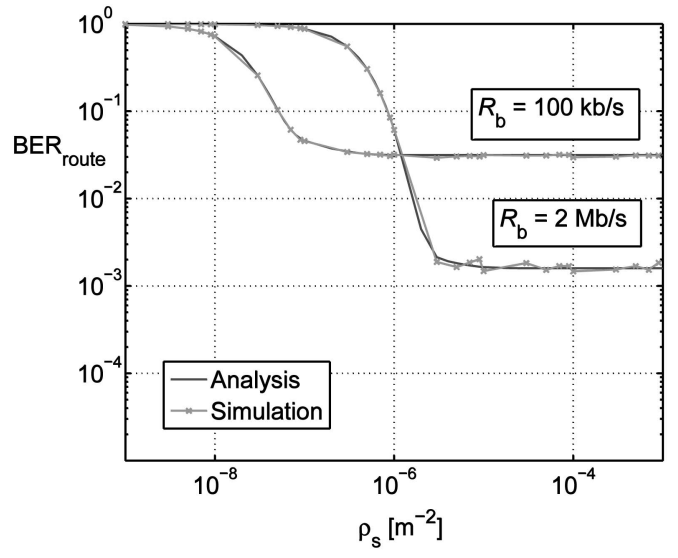

Fig. 5. Route BER as a function of node spatial density. Both analytical results and simulation results are compared.

signals come from the nodes in the first tier around the receiver, and interference from nodes in other tiers are not significant. In Fig. 4, we compare the route BER in two scenarios: 1) interference signals are from nodes in the first tier only and 2) interference signals are from nodes in both the first tier and the second tier. The results are obtained assuming that the number of nodes is $N=289$, the transmit power is $P_{\mathrm{t}}=1 \mathrm{mw}$, the packet size is $L=1,000 \mathrm{~b} / \mathrm{pck}$, and the packet transmission rate is $\lambda_{\mathrm{t}}=0.5 \mathrm{pck} / \mathrm{s}$. The considered data rates are $R_{\mathrm{b}}=2 \mathrm{Mb} / \mathrm{s}$ and $R_{\mathrm{b}}=100 \mathrm{~kb} / \mathrm{s}$. It can be observed that the route BER in scenarios 1) and 2) are not significantly different for both data-rate values. As a result, without significant loss of accuracy, one can estimate the route BER by considering only the interference from nodes in the first tier.

To verify our analysis, we also compute the route BER via Monte-Carlo simulations. In the simulation, each node transmits packets according to the RESGO MAC protocol. For each bit received by the receiver, the detection decision is made based on the total received signal (i.e., the signal from the transmitter, the interference signals, and the thermal noise). If the received bit is different from the original bit sent by the transmitter, it is declared as a bit error. Through a Monte-Carlo approach, the link BER can then be obtained by calculating the ratio between the number of bits received in error and the total number of bits received. Finally, the route BER can be obtained from the link BER using (21). In Fig. 5, we compare the route BER obtained from analysis with that obtained by simulation. The parameters used are the same as those in Fig. 4. It can be observed that both analytical and simulation results are in good agreement.

\subsubsection{Approximate Analysis}

The link BER and the route BER can be rigorously evaluated with the approach presented in Section 4.1.1. However, in this section, we provide an approximation approach which allows us to calculate the link BER and the route BER more conveniently. In this approach, the link BER is evaluated by assuming that, in each link, the total interference signal can be modeled as a Gaussian process 
with variance corresponding to the total average interference power. While we used this approach in our earlier works [10], [23], a more accurate analysis reveals that this approach has limited validity. We now show how to correctly use this approach. In order to do this, we first evaluate the average interference power as observed by the receiver. Since each node transmits with a common power $P_{\mathrm{t}}$, if all nodes simultaneously transmit, it can be shown that the total interference power experienced by the receiver at the center of the network is [17]

$$
P_{\text {total }}^{\text {grid }}=\alpha P_{\mathrm{t}} I_{\text {grid }}
$$

where

$$
I_{\text {grid }} \triangleq \frac{1}{r_{\text {link }}^{\gamma}} \sum_{i=1}^{i_{\max }}\left[\frac{4}{i^{\gamma}}+\frac{4}{(\sqrt{2} i)^{\gamma}}+\sum_{j=1}^{i-1} \frac{8}{\left(\sqrt{i^{2}+j^{2}}\right)^{\gamma}}-1\right] .
$$

The first term in the summation corresponds to the four nodes in each tier that are aligned with the receiver at the center of the network, the second term in the summation corresponds to contribution from the four nodes at the corners of each tier at distance $\sqrt{2} i r_{\text {link }}$ from the receiver, and, finally, the last term in the summation corresponds to the contribution of all the other nodes in each tier. Note that, in each tier, there are eight nodes at distance $\sqrt{i^{2}+j^{2}} r_{\text {link }}$ from the receiver, for $j<i$.

With the RESGO MAC protocol, each node transmits with probability $p_{\text {tran }}$ and, thus, the average interference power can be written as

$$
\mathrm{E}\left[P_{\text {int }}^{\text {grid }}\right]=p_{\text {tran }} P_{\text {total }}^{\text {grid }}=\left(1-e^{-\frac{\lambda_{\mathrm{t}} L}{R_{\mathrm{b}}}}\right) P_{\text {total }}^{\text {grid }} .
$$

Considering BPSK signaling and assuming that the interfering noise is Gaussian, the BER on each link of a route can be written as [24]

$$
\mathrm{BER}_{\text {link }_{\text {grid }}}=Q\left(\sqrt{\frac{2 \alpha P_{\mathrm{t}} / r_{\text {link }}^{\gamma}}{P_{\text {thermal }}+\mathrm{E}\left[P_{\text {int }}^{\text {grid }}\right]}}\right) .
$$

The route BER can then be evaluated using (21).

The route BER from the exact interference analysis presented in Section 4.1.1 shows the existence of a BER floor for increasing values of the node spatial density or transmit power. A typical BER floor can be observed in Fig. 5. This is intuitively expected because, after the network becomes dense enough, increasing the node spatial density (i.e., reducing the hop length) no longer improves the link SNR, as the interfering nodes also become close enough to the receiver. However, the route BER predicted by the Gaussian assumption for the interference signal presents a much lower BER floor. Via a careful analysis, the route BER floor can be approximated with the following expression:

$$
\mathrm{BER}_{\text {floor }} \approx \frac{3 \bar{n}_{\text {grid }} \lambda_{\mathrm{t}} L}{4 R_{\mathrm{b}}} .
$$

Motivated by the above considerations, one finds out that an accurate approximate expression for the route BER can be written as

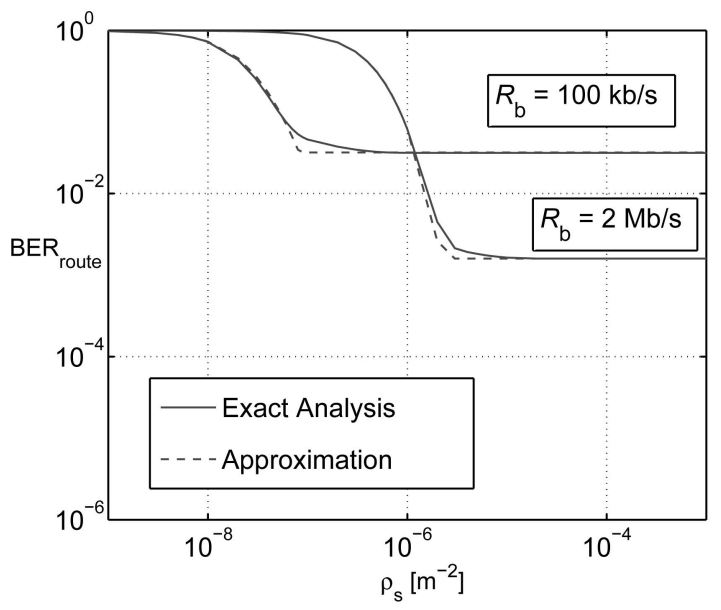

Fig. 6. A comparison between the route BER obtained with the exact interference analysis and the approximation.

$\operatorname{BER}_{\text {route }} \simeq$
$\max \left\{\left(1-\left[1-Q\left(\sqrt{\frac{2 \alpha P_{\mathrm{t}} / r_{\text {link }}^{\gamma}}{P_{\text {thermal }}+\mathrm{E}\left[P_{\text {int }}^{\text {grid }}\right]}}\right)\right]^{\bar{n}_{\text {grid }}}\right), \frac{3 \bar{n}_{\text {grid }} \lambda_{\mathrm{t}} L}{4 R_{\mathrm{b}}}\right\}$.

In Fig. 6, to verify the accuracy of this approximation, we compare the route BER obtained from the exact interference analysis (e.g., using the approach in Section 4.1.1) with that obtained from the approximate analysis (e.g., using (27)) for two different values of data rate. The parameters used are the same as those in Fig. 4. It can be observed that the route BER obtained from the approximation closely matches the route BER obtained from the exact interference analysis.

\subsection{Random Topology}

In the case of random topology, it is difficult to obtain exact analytical expressions for the link BER and the route BER. Therefore, we resort to a simulation approach for the evaluation of the BER. For each communication link, a transmitter and receiver pair is generated. The distance between the transmitter and the receiver (i.e., $W$ ) is randomly generated from the hop length distribution given in (4). Then, a number of interfering nodes within the circle of radius $2 W$ centered at the receiver are generated according to a two-dimensional Poisson distribution. Their positions are uniformly distributed. For each node, we schedule transmissions of packets according to the RESGO MAC protocol. For each bit received at the receiver, a threshold-based decision is made. The detected bit is declared as an error if it differs from the original bit sent by the transmitter. The link BER is obtained by computing the ratio of the number of erroneous bits and the total number of received bits. To obtain the route BER, we generate a route with $\bar{n}_{\text {rand }}$ links and compute the route BER as follows:

$$
\mathrm{BER}_{\text {route }}^{\mathrm{rand}}=1-\prod_{i=1}^{\bar{n}_{\mathrm{rand}}} \mathrm{BER}_{\text {link } i}
$$

where $\mathrm{BER}_{\text {link } i}$ is the BER of the $i$ th link of a route. The average route BER is then obtained by computing the 
arithmetic average of a large number of realizations of $\mathrm{BER}_{\text {route }}^{\text {rand }}$.

\section{Optimal Common transmit Power}

In this section, we derive the optimal common transmit power for ad hoc wireless networks with grid and random topologies, respectively.

\subsection{Optimal Common Transmit Power for Networks with Square Grid Topology}

With the exact BER analyzed in Section 4.1.1, one can always numerically find the minimum transmit power which would satisfy the desired BER threshold, provided that the BER threshold is above the BER floor. However, it is more insightful to derive an analytical expression for the optimal transmit power. In this section, we will derive an expression for the optimal transmit power for a given maximum tolerable route BER from the approximate route BER expression given in (27).

In a network with grid topology, the common transmit power used by each node should be large enough so that the BER at the end of a multihop route with an average number of hops $\bar{n}_{\text {grid, }}$ given by (5), is lower than the maximum tolerable value, denoted as $\mathrm{BER}_{\mathrm{th}}$. If the required $\mathrm{BER}_{\mathrm{th}}$ is higher than $\mathrm{BER}_{\mathrm{floor}}$, there exists a power which can satisfy this requirement. This transmit power is such that the following inequality must be satisfied:

$$
1-\left(1-\mathrm{BER}_{\text {link }_{\text {grid }}}\right)^{\bar{n}_{\text {grid }}} \leq \mathrm{BER}_{\text {th }} .
$$

Substituting expression (25) for $\mathrm{BER}_{\text {link }_{\text {grid }}}$ into (29), one can rewrite (29) as

$$
1-\left[1-Q\left(\sqrt{\frac{2 \alpha P_{\mathrm{t}} / r_{\text {link }}^{\gamma}}{P_{\text {thermal }}+\mathrm{E}\left[P_{\text {int }}^{\text {grid }}\right]}}\right)\right]^{\bar{n}_{\text {grid }}} \leq \mathrm{BER}_{\text {th }} .
$$

Substituting $F k T_{0} R_{\mathrm{b}}$ for $P_{\text {thermal, }}$, the expression given in (24) for $\mathrm{E}\left[P_{\text {int }}^{\mathrm{grid}}\right]$, and rewriting (30) in terms of $P_{\mathrm{t}}$, one obtains the following expression for the optimal transmit power:

$$
\begin{aligned}
P_{\mathrm{t}}^{*}= & F k T_{0} R_{\mathrm{b}}\left[\frac{2 \alpha}{r_{\text {link }}^{\gamma} \Psi}-\alpha\left(1-e^{-\frac{\lambda_{\mathrm{t}} L}{R_{\mathrm{b}}}}\right) I_{\text {grid }}\right]^{-1} \\
& (\text { dimension : [Watt }]),
\end{aligned}
$$

where

$$
\Psi \triangleq\left\{Q^{-1}\left[1-\left(1-\mathrm{BER}_{\mathrm{th}}\right)^{1 / \bar{n}_{\text {grid }}}\right]\right\}^{2}
$$

and $I_{\text {grid }}$ is given by (23). The expression given in (31) corresponds to the optimal transmit power when

$$
\mathrm{BER}_{\text {floor }} \leq \mathrm{BER}_{\mathrm{th}}
$$

for a given data rate $R_{\mathrm{b}}$, node spatial density $\left(r_{\text {link }} \simeq 1 / \sqrt{\rho_{\mathrm{s}}}\right)$, number of nodes in the network $\left(\bar{n}_{\text {grid }} \simeq \sqrt{N} / 2\right)$, antenna gains, and carrier frequency ( $\alpha$ depends on them).

\subsection{Optimal Common Transmit Power for Networks with Random Topology}

In a network with two-dimensional Poisson node distribution, the hop length is random. Consequently, it is difficult to find a closed-form expression for the optimal transmit power. In this case, we resort to simulations. For a given data rate and transmit power, we compute the average route BER with the approach described in Section 4.2. The same process is repeated for different combinations of data rate and transmit power. At the end, we thus obtain the average route BERs for different combinations of data rate and transmit power, and these are used to obtain contours of power-data rate pairs in correspondence to which the route $B E R$ is equal to maximum tolerable $B_{\text {th }}$ values.

\section{Performance Metrics}

\subsection{Node and Network Lifetime}

Network lifetime is an important performance indicator for wireless ad hoc and sensor networks. There are several definitions of network lifetime. While network lifetime is defined as the time to the first node failure in [25] and [26], it is defined in terms of the fraction of surviving nodes in the network in [27]. In this paper, following [25] and [26], we consider the time to the first node failure as the network lifetime (i.e., a worst-case approach).

In this section, we present a simple analysis for computing the average lifetime of a node. We assume that the RESGO MAC protocol is used and every node has an initial finite battery energy denoted by $E_{\text {batt }}$. For a given data rate $R_{\mathrm{b}}$, the time taken to transmit one packet is $L / R_{\mathrm{b}}$. Therefore, the total amount of energy consumed per transmitted packet can be written as

$$
\left.E_{\text {packet }}=P_{\mathrm{t}} \times \frac{L}{R_{\mathrm{b}}}(\text { dimension : [Joules }]\right) .
$$

Since packets are transmitted with average rate $\lambda_{t}$, the average energy depleted per second is simply $\lambda_{\mathrm{t}} E_{\text {packet }}$. Finally, the total time it takes to completely exhaust the initial battery energy can be written as

$$
\left.\tau=\frac{E_{\text {batt }}}{\lambda_{\mathrm{t}} E_{\text {packet }}}=\frac{E_{\text {batt }} R_{\mathrm{b}}}{\lambda_{\mathrm{t}} L P_{\mathrm{t}}} \quad(\text { dimension : [sec }]\right) .
$$

Note that, due to uniform traffic assumption (i.e., all nodes generate approximately the same amount of traffic load), on average, all nodes exhaust their battery at the same time. In addition, this simple analysis does not take into account the energy consumed when a node is receiving and processing packets. In reality, the lifetime of a node will be shorter than what is predicted by our analysis. Recent studies have shown that the energy consumption ratio when a device is in idle mode, receiving mode, and transmitting mode is 1:1.2:1.68 (see, e.g., [28]). Therefore, transmitting is the most "expensive" (from a battery consumption perspective) activity. Power consumption in idle and receiving modes can be taken into account by properly extending the proposed approach.

\subsection{Effective Transport Capacity}

The effective transport capacity (dimension: $[\mathrm{b}-\mathrm{m} / \mathrm{s}]$ ) is an important wireless network performance metric which simultaneously captures the amount of information generated and the distance over which it can be transported in the network [23]. As is evident from (21), the BER at the end 
of a multihop route increases as the distance traversed by an information bit increases, i.e., as the number of hops increases. Consequently, the distance that an information bit can traverse while maintaining sufficiently low BER may be less than the length of a multihop route with an average number of hops (i.e., a message could reach the destination, but the final BER could be so high that the destination could not decode the received message correctly). The following QoS condition, in terms of tolerable BER at the end of a multihop route with $n$ hops, formalizes the requirement of sufficiently low route BER:

$$
\mathrm{BER}_{\text {route }}^{(n)} \leq \mathrm{BER}_{\text {th }},
$$

where $\mathrm{BER}_{\text {route }}^{(n)}$ is the BER at the end of a $n$-hop route. We refer to the distance which a bit can traverse, while satisfying a desired BER QoS condition, as maximum sustainable distance. The effective transport capacity of a single route can then be defined as

$$
C_{\mathrm{Te}}^{\mathrm{sr}} \triangleq \lambda_{\mathrm{t}} \times L \times \min \left(d_{\mathrm{s}}, d_{\mathrm{a}}\right),
$$

where $d_{\mathrm{s}}$ is the maximum sustainable distance and $d_{\mathrm{a}}$ is the distance associated with an average multihop route. Note that, in (36), the traffic transmission rate is used, whereas one should consider a traffic generation rate which represents the average rate of information flowing in a route. However, since we have assumed in this paper that a node always has packets to send, the average flow rate in a route, in this case, is equal to the average transmission rate $\lambda_{\mathrm{t}}$. The value $\min \left(d_{\mathrm{s}}, d_{\mathrm{a}}\right)$ can be interpreted as the average sustainable distance. It represents the distance that a bit, on average, can traverse. A fundamental underlying assumption in (36) is that only the source node of a route contributes "effective" information (on average, a source node transmits $\lambda_{\mathrm{t}} L$ bits every second). In this sense, the intermediate nodes act as relay nodes, but they do not contribute to the effective transport capacity in terms of information bits. However, relay nodes do contribute to the effective transport capacity in the sense of increasing the distance traversed by each bit transmitted by the source.

In this paper, the effective transport capacity of the network is obtained by adding the single-route effective transport capacities of all the disjoint routes in the network. ${ }^{4}$ Assuming that each route has an average number of hops, the number of disjoint routes corresponding to a scenario where each node belongs to a particular route (either as the source, the destination, or a relay) in networks with grid and random topologies can be estimated as $N / \bar{n}_{\text {grid }}$ and $N / \bar{n}_{\text {rand, }}$ respectively. The extension of the proposed analysis to a scenario where multihop routes can cross can be done by following the approach in [29].

In the following section, we derive analytical expressions for $d_{\mathrm{s}}$ and $d_{\mathrm{a}}$ in networks with square grid topology and networks with two-dimensional Poisson topology, respectively. The corresponding effective transport capacities in both cases can be obtained using (36).

4. The disjoint routes assumption is particularly assumed here for simplicity in calculating the network's effective transport capacity. However, other results derived in this paper hold regardless of whether routes are disjoint or not.

\subsubsection{Square Grid Topology}

First, we compute the maximum sustainable distance. In the case of square grid topology, the route BER at the end of a generic $n$-hop route can be obtained by replacing $\bar{n}_{\text {grid }}$ in (27) with $n$. Thus, (35) will be satisfied if

$\max \left\{\left(1-\left[1-Q\left(\sqrt{\frac{2 \alpha P_{\mathrm{t}} / r_{\text {link }}^{\gamma}}{P_{\text {thermal }}+\mathrm{E}\left[P_{\text {int }}^{\text {grid }}\right]}}\right)\right]^{n}\right), \frac{3 n \lambda_{\mathrm{t}} L}{4 R_{\mathrm{b}}}\right\} \leq \mathrm{BER}_{\mathrm{th}}$.

Solving (27) for $n$, the maximum sustainable number of hops can be written as

$$
n_{\mathrm{s}}^{\text {grid }}=\min \left\{\frac{\ln \left(1-\mathrm{BER}_{\mathrm{th}}\right)}{\ln \left(1-\mathrm{BER}_{\text {link }_{\text {grid }}}\right)}, \frac{4 R_{\mathrm{b}} \mathrm{BER}_{\mathrm{th}}}{3 \lambda_{\mathrm{t}} L}\right\} .
$$

Since all hops in a network with grid topology have the same length $r_{\text {link, }}$ the maximum sustainable distance is simply the product of the maximum sustainable number of hops and the hop length:

$$
d_{\mathrm{s}}^{\text {grid }}=n_{\mathrm{s}}^{\text {grid }} r_{\text {link }}
$$

Similarly, the average route distance in a grid topology, i.e., $d_{\mathrm{a}}^{\text {grid }}$, can be obtained as the product of the average number of hops $\bar{n}_{\text {grid }}$ with the hop length $r_{\text {link }}$, i.e., $d_{\mathrm{a}}^{\text {grid }}=\bar{n}_{\text {grid }} r_{\text {link }}$.

\subsubsection{Random Topology}

As in the case of a network with grid topology, in order to obtain the single-route (and the aggregate) effective transport capacity of a network with random topology, we need to compute the maximum sustainable distance and the average route distance. In this case, however, the maximum sustainable distance is obtained via computer simulations. We construct a route by recursively generating links whose lengths are obtained according to the CDF given in (4). After the generation of a link, the route BER is then computed and compared with the threshold value, i.e., $\mathrm{BER}_{\mathrm{th}}$. If the BER is lower than $\mathrm{BER}_{\text {th }}$, a new link will be generated and added to the route. This procedure is repeated until the route BER exceeds $\mathrm{BER}_{\mathrm{th}}$. The maximum sustainable distance is obtained by adding the lengths of the links of the formed multihop route. A sufficiently large number of multihop routes are generated and the average sustainable distance is computed by averaging over the maximum sustainable distances corresponding to the route realizations.

What now remains to be computed is the average route distance in a random topology, i.e., $d_{\mathrm{a}}^{\text {rand }}$. The average number of hops in a route between a source and a destination is $\bar{n}_{\text {rand }}$. To find the average distance, one can compute the expected value of the route distance in a $\bar{n}_{\text {rand }}$-hop route, that is,

$$
d_{\mathrm{a}}^{\mathrm{rand}}=\mathrm{E}\left[\sum_{i=1}^{\bar{n}_{\text {rand }}} W_{i}\right]
$$

where $W_{i}$ is the hop length of the $i$ th link. Since $\bar{n}_{\text {rand }}$ is a deterministic quantity, (40) can be simplified to

$$
d_{\mathrm{a}}^{\mathrm{rand}}=\bar{n}_{\mathrm{rand}} \mathrm{E}[W] .
$$


TABLE 1

Major Network Parameter Values Used in Considered Networking Scenarios

\begin{tabular}{|l|c|}
\hline Parameters & Values \\
\hline \hline Number of nodes in the network $(N)$ & 289 nodes \\
\hline Node spatial density $\left(\rho_{\mathrm{s}}\right)$ & $10^{-7} \mathrm{~m}^{-2}$ \\
\hline Packet length $(L)$ & $10^{3} \mathrm{bits}$ \\
\hline Packet arrival rate at each node $(\lambda)$ & $0.5 \mathrm{pck} / \mathrm{s}$ \\
\hline Pathloss exponent $(\gamma)$ & 2 \\
\hline Carrier frequency $\left(f_{\mathrm{c}}\right)$ & $2.4 \mathrm{GHz}$ \\
\hline Room temperature $\left(T_{0}\right)$ & $300 \mathrm{~K}$ \\
\hline Noise figure $(F)$ & $6 \mathrm{~dB}$ \\
\hline
\end{tabular}

\section{Results ANd Discussion}

Numerical results, along with their implications, are presented and discussed in this section. The values of the major network parameters are given in Table 1 unless specified otherwise.

\subsection{Optimal Transmit Power and Data Rate}

In Fig. 7, the optimal common transmit power is shown as a function of the data rate. The optimal power-data rate curves are shown for various values of $\mathrm{BER}_{\mathrm{th}}$. For each case of $\mathrm{BER}_{\mathrm{th}}$, both the optimal transmit power computed numerically with the exact interference analysis and the optimal transmit power computed with the approximation given in (31) are shown. It can be observed that the optimal transmit power increases as the data rate increases. This can be explained as follows: Although transmitting packets at a higher data rate reduces the vulnerable time (and, hence, smaller interference), increasing the data rate (i.e., bandwidth) also increases the thermal noise. Therefore, the minimum transmit power required to sustain the network connectivity has to increase accordingly. We also observe a close match between the optimal transmit power predicted

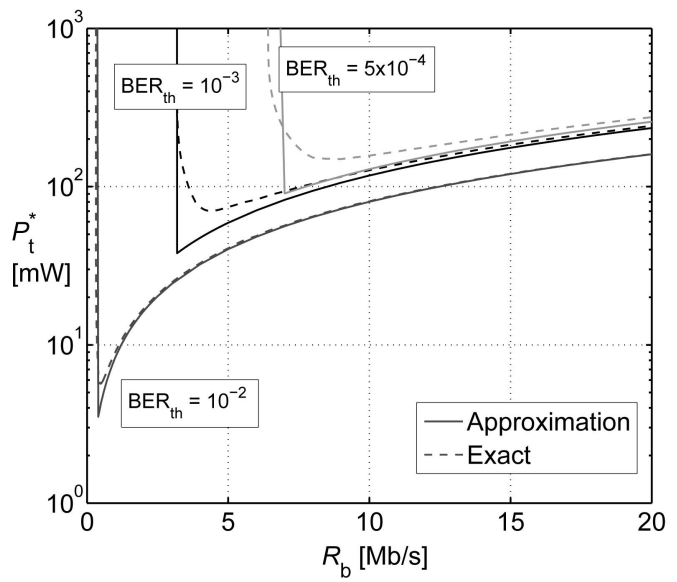

Fig. 7. Optimal common transmit power in a network with square grid topology. Only interference from nodes in the first tier around the receiver is considered.

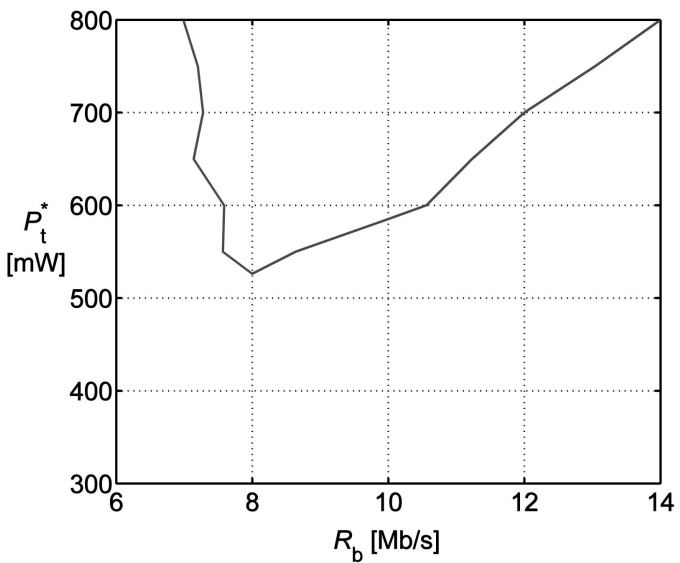

Fig. 8. Optimal common transmit power in a network with random topology and $\mathrm{BER}_{\mathrm{th}}=10^{-3}$.

with the approximation and the one obtained numerically from the exact interference analysis.

In addition, it can be observed from Fig. 7 that there is a critical data rate, beyond which the desired $\mathrm{BER}_{\text {th }}$ cannot be satisfied for any transmit power. The critical data rate occurs at the point where the $\mathrm{BER}_{\text {floor }}$ for that particular data rate becomes higher than the desired BER $\mathrm{Bt}_{\mathrm{th}}$. Consequently, for an uncoded transmission and without retransmission, no transmit power can achieve the desired BER threshold.

The optimal transmit power is also minimized at the data rate near the critical point. This suggests that the data rate also plays an important role in the design of wireless ad hoc and sensor networks-that is, for a given node spatial density, if the data rate is carefully chosen, the transmit power can be minimized, prolonging the network's lifetime.

A scenario with random topology is shown in Fig. 8 for the case where $\mathrm{BER}_{\mathrm{th}}=10^{-3}$. Similar to the case of grid topology, the optimal transmit power increases as the data rate increases. However, in the case of random topology, as expected, a much higher transmit power is required to sustain connectivity. This is due to the fact that the hop length is random. In other words, a long hop is likely to be present in a multihop route, and this significantly reduces the route BER [15]. In addition, in the case of random topology, higher data rates are required to support connectivity.

The optimal transmit power shown in Fig. 7 is computed based on the assumption that there is no packet retransmission on each link of a route. In some applications, packet retransmission may also be used. In Fig. 9, we compare the optimal transmit power in three different scenarios: 1) no retransmissions on each link, 2) one retransmission is allowed on each link, and 3) five retransmissions are allowed on each link. The BER th $_{\text {ch }}$ considered in this case is $10^{-3}$. It can be observed that allowing retransmission can decrease the minimum transmit power required to keep the network connected. This is because retransmission improves the BER and, thus, a lower transmit power is required to get a packet to the destination with the desired quality. It can also be observed that the critical data rate shifts leftward as the number of retransmissions increase. Thus, by allowing retransmission, the network connectivity can be supported at lower data rates. 


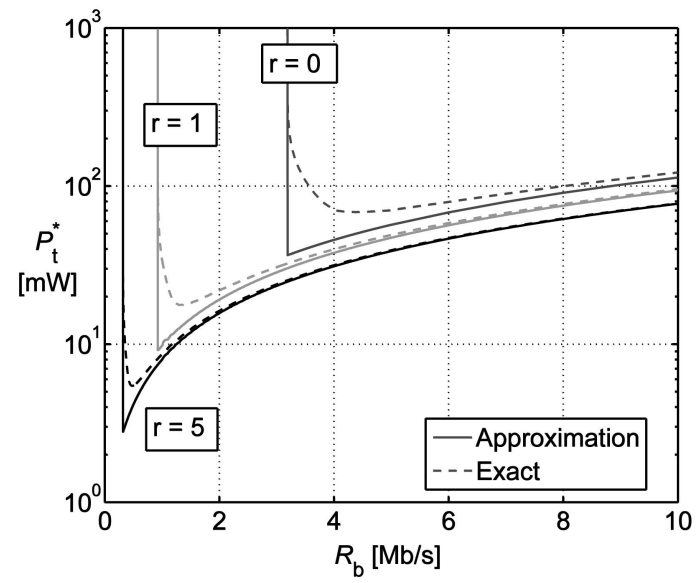

Fig. 9. Optimal common transmit power in a network with square grid topology and $\mathrm{BER}_{\mathrm{th}}=10^{-3}$. Different number of retransmissions are compared.

\subsection{Optimal Transmit Power and Node Spatial Density}

Fig. 10 illustrates the optimal transmit power as a function of the node spatial density. In this scenario, the data rate is fixed at $R_{\mathrm{b}}=4 \mathrm{Mb} / \mathrm{s}$, and the considered BER $\mathrm{Bth}_{\text {th }}$ values are $10^{-2}$ and $10^{-3}$, respectively. Both the optimal transmit power computed numerically with the exact interference analysis and the optimal transmit power computed with the approximation given in (31) are compared. It can be observed that the optimal transmit power decreases linearly in the log scale as the node spatial density increases. This can be explained as follows: When the network is sparse (i.e., low node spatial density), the minimum transmit power has to be increased in order to preserve connectivity. However, as the network becomes denser, the nodes are closer to their neighbors and, therefore, the power required to sustain connectivity decreases.

Note that the $B E R_{\text {floor }}$ for the scenario considered in Fig. 10 is fixed because the data rate $R_{\mathrm{b}}$, the number of nodes $N$, the traffic transmission rate $\lambda_{\mathrm{t}}$, and the packet length $L$ are fixed. As long as the desired BER $\mathrm{Bth}_{\text {h }}$ is greater than this $\mathrm{BER}_{\text {floor }}$, there is always a minimum power to sustain the connectivity at any node spatial density. On the

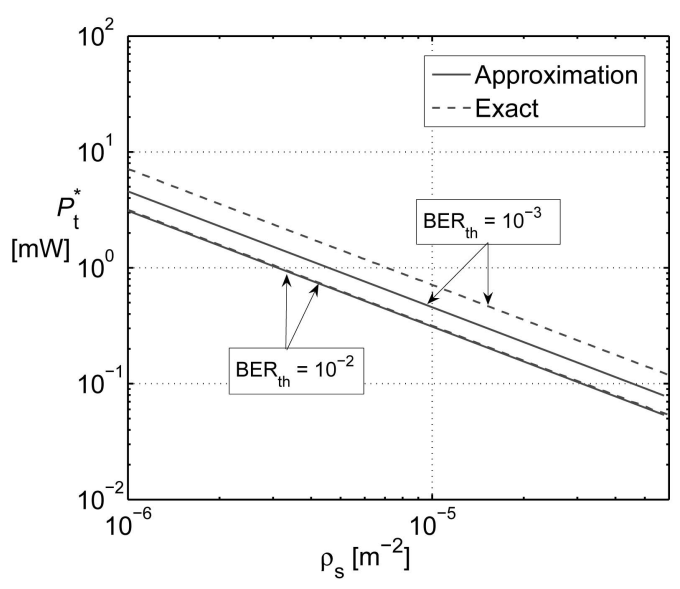

Fig. 10. Optimal common transmit power in a network with grid topology as a function of node spatial density.

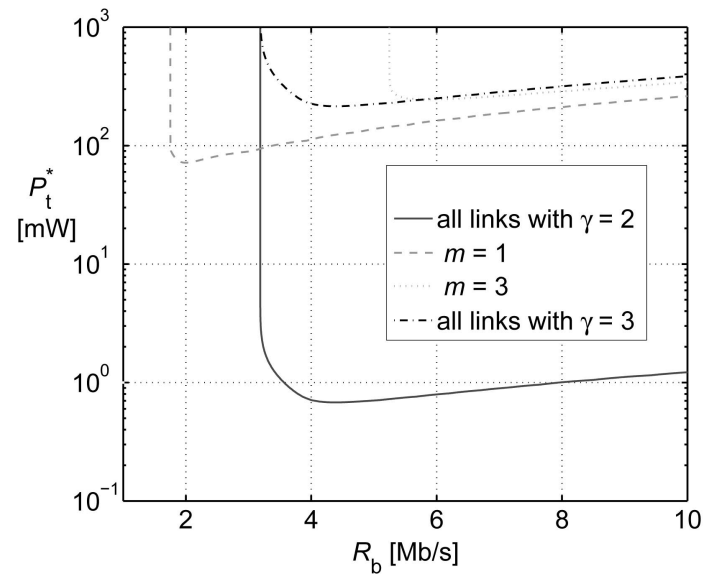

Fig. 11. Optimal common transmit power in a network with square grid topology for various configurations of the links' pathloss exponents. In this case, the node spatial density is $\rho_{\mathrm{s}}=10^{-5} \mathrm{~m}^{-2}$ and $\mathrm{BER}_{\mathrm{th}}=10^{-3}$.

other hand, in the case where BER th is less than the $\mathrm{BER}_{\text {floor }}$, no transmit power can satisfy the desired connectivity criteria at any node spatial density, unless forward error correction or retransmission techniques are used.

\subsection{Effects of Strong Propagation Pathloss}

In a realistic communication environment, some links may experience higher pathloss than others due to impairments such as shadowing [20]. In order to study how pathloss affects the optimal common transmit power, next, we consider scenarios where some links in a multihop route are characterized by higher pathloss than the others. We study the impact of these high pathloss links on the optimal transmit power by varying the number of these links in a multihop route with an average number of hops.

Fig. 11 illustrates the optimal common transmit power as a function of the data rate in a scenario where the RESGO MAC protocol is used and the network topology is a square grid. Four scenarios are considered:

1. all links in an average multihop route are characterized by a pathloss exponent $\gamma=2$,

2. the $m=1$ link is characterized by a pathloss exponent $\gamma=3$, whereas the other links in the route are still characterized by $\gamma=2$,

3. the $m=3$ links are characterized by a pathloss exponent $\gamma=3$, whereas the other links in the route are still characterized by $\gamma=2$, and

4. all links in the route are characterized by a pathloss exponent $\gamma=3$.

In the scenarios where the pathloss exponents for different links are not the same, attenuation of the signals originating from interfering nodes will also vary. If the pathloss for each interfering node, relative to the receiver, were known, then the exact interference power could be computed. However, this would only correspond to a specific realization of the network, where one needs to determine the propagation loss on each individual link from any node to any other nodes.

In order to estimate the interference power, we assume, for simplicity, that the interference power from interfering 
nodes attenuate with a common pathloss exponent $\gamma_{c}$, given by the following heuristic expression:

$$
\gamma_{\mathrm{c}}=\frac{\bar{n}_{\text {grid }}-m}{\bar{n}_{\text {grid }}} 2+\frac{m}{\bar{n}_{\text {grid }}} 3 .
$$

In other words, (42) for the common pathloss exponent corresponds to a weighted average between 2 and 3 . The intuition behind (42) is that, if the number of links per route with $\gamma=2$ is large, then the pathloss exponent of each interfering node to the receiver should be close to 2; on the other hand, if the number of links with $\gamma=3$ is large, then the pathloss exponent of each interfering node to the receiver should be close to 3 . We emphasize that the expression in (42) is only a heuristic. However, the results obtained with this analysis provide, at least trendwise, meaningful insights.

Generally, it can be observed from Fig. 11 that the power required to sustain network connectivity increases as the number of links with $\gamma=3$ increases. Moreover, the transmit power has to be increased substantially even if only one link in a multihop route experiences higher pathloss than the others. This has a significant implication. It suggests that the use of a common transmit power will work well in situations where each link experiences relatively the same level of propagation loss. If it is highly likely that at least one link per route is going to experience unusually high pathloss, then it is not efficient to use a common transmit power because all nodes have to significantly raise their transmit power to recover from the degradation caused by a few high pathloss links. A possible solution to this problem is to modify the routing protocol in such a way that, at the moment of route creation, link quality is selected as one of the routing criteria [30], [31]. With this capability, a routing protocol may be able to select a multihop route which bypasses high pathloss links. Another potential solution is obviously considering local power control [4], [5], [6]. These possible extensions are currently under investigation.

\subsection{Connectivity Robustness to Node Spatial Density Changes}

The optimal transmit power analyzed earlier is the minimum transmit power, which is sufficient to maintain network connectivity at a particular node spatial density. In some situations, the node spatial density of a network can change over time, for example, when nodes join/leave the network or when the batteries of some nodes are exhausted. A network can lose connectivity immediately if the node spatial density is lower than the initial value for which the power is optimally chosen. In order to provide robustness against a possible node spatial density reduction, nodes may transmit at a power level higher than the required minimum level. In this section, we evaluate the connectivity robustness as the network becomes sparser. We define the initial density at which the network starts forming as $\rho_{\mathrm{s}_{0}} \triangleq N_{0} / A$, where $N_{0}$ is the initial number of nodes in the network. Keeping the network area constant, the node spatial density decreases if the number of nodes becomes lower than $N_{0} .{ }^{5}$ We denote the

5. The proposed analysis can be straightforwardly extended to a scenario where the number of nodes is fixed and the network area increases.

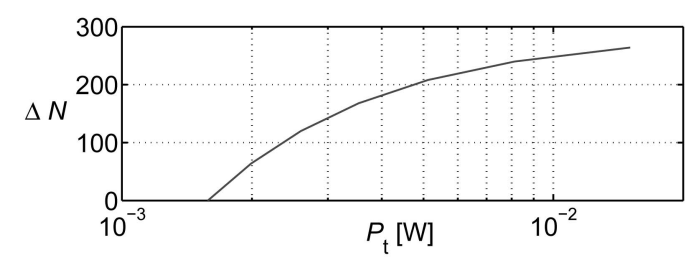

(a)

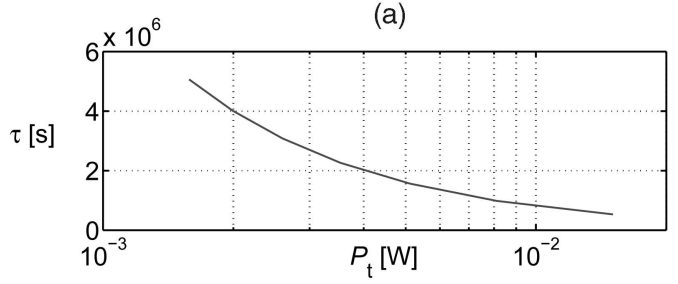

(b)

Fig. 12. (a) Increase in connectivity level and (b) lifetime of a node as functions of transmit power in a network with grid topology.

decrease in the number of nodes as $\Delta N \triangleq N_{0}-N_{\mathrm{f}}$, where $N_{\mathrm{f}}$ is the final number of nodes after some nodes disappear from the network-some nodes could either move out of the network or their batteries could be exhausted.

Fig. 12a shows a plot of $\Delta N$ as a function of the transmit power. In this scenario, the initial number of nodes is $N_{0}=289$ and the network surface area is $A=10^{8} \mathrm{~m}^{2}$. The minimum transmit power required to sustain the network connectivity for this initial node spatial density is $P_{\mathrm{t}_{0}}^{*}=1.6 \mathrm{~mW}$. In this figure, for each transmit power, the node spatial density and the number of nodes $N_{\mathrm{f}}$ at which the network can still maintain connectivity is calculated.

This allows one to obtain $\Delta N$, which, in this figure, can be interpreted as the connectivity robustness against a change in terms of node spatial density. For example, $\Delta N=$ 100 indicates that a network can still maintain connectivity even if 100 nodes disappear.

It can be observed from Fig. 12a that, as the transmit power increases, $\Delta N$ increases. ${ }^{6}$ This is intuitively expected because, for higher transmit power, the network can tolerate lower node spatial densities, i.e., it can work even if the network becomes sparser. However, as the transmit power level increases, the rate of gain, in terms of connectivity robustness, asymptotically decreases.

While connectivity robustness can be increased with higher transmit power, this comes at the expense of the nodes' and network's lifetimes. Fig. 12b illustrates the behavior of the lifetime of a node as a function of the transmit power. In particular, the lifetime of a node is given by (34). As expected, the node lifetime decreases as the transmit power increases to support communication in a sparser network.

6. The decrease in the number of nodes $(\Delta N)$ is computed as if the nodes are still in a square grid topology after $\Delta N$ nodes disappear from the network. In reality, nodes will probably disappear from the network in a random fashion, and the topology is likely to be random after some nodes disappear. Thus, the power required to maintain network connectivity is expected to be much higher than what is shown in Fig. 12. However, the results shown in Fig. 12 provide a meaningful insight in terms of the trend involved. 


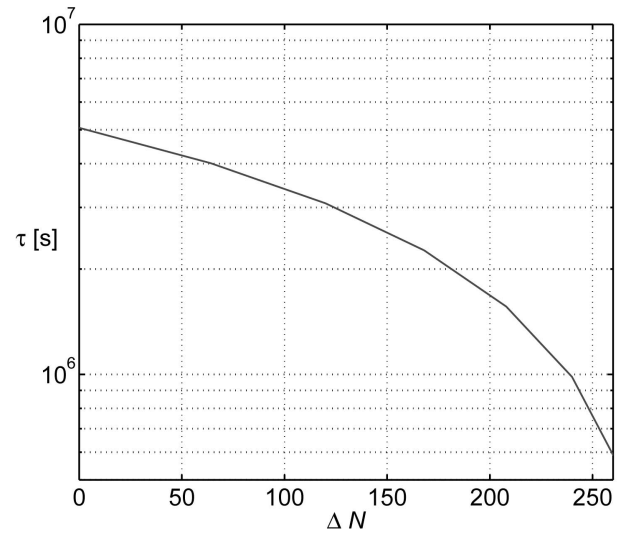

Fig. 13. Lifetime of a node as a function of connectivity.

As shown in Fig. 12a and Fig. 12b, it is clear that there is a trade-off between connectivity increase and nodes' longevity. This trade-off is clearly shown in Fig. 13, where network lifetime is shown as a function of the connectivity robustness. It can be observed that the slope of the curve becomes steeper as $\Delta N$ increases. This suggests that trading network lifetime for connectivity is beneficial when the level of connectivity is small; however, the benefit decreases at high values of $\Delta N$. In other words, to have high protection against node spatial density changes, significant network lifetime has to be sacrificed.

The increased robustness against node spatial density reduction is also exhibited by the behavior of the effective transport capacity. Fig. 14 shows the behavior of the effective transport capacity, in a scenario with square grid topology, as a function of the number of nodes. The results are obtained assuming that the data rate is $R_{\mathrm{b}}=4 \mathrm{Mb} / \mathrm{s}$, the network area is $A=10^{8} \mathrm{~m}^{2}$, and the desired level of BER is $\mathrm{BER}_{\text {th }}=10^{-3}$. By keeping the area constant, an increase of the number of nodes corresponds to an increase of the node spatial density. Three levels of transmit powers are considered: 1) $\left.P_{\mathrm{t}_{0}}^{*}=1.6 \mathrm{~mW}, 2\right) 2 \times P_{\mathrm{t}_{0}}^{*}$, and 3) $5 \times P_{\mathrm{t}_{0}}^{*}$.

Considering the effective transport capacity curve, one is mostly interested in the region where the network is connected. This is the region where an information bit can be transported over an average route while satisfying the QoS constraint on the maximum tolerable BER, i.e., BER $_{\text {th }}$ [23]. The solid curve in Fig. 14 indicates the effective transport capacity in the region where the network has connectivity. The connectivity region for each considered transmit power starts from the point where each dashed curve reaches the solid curve and ends at the knee of the curve (e.g., around $N=425$ ). It can be seen from Fig. 14 that, when the transmit power is increased from $P_{t_{0}}^{*}$ to $2 \times P_{\mathrm{t}_{0}}^{*}$, the connectivity region of the network increases. More specifically, the network gains connectivity at $N=$ 289 in the case where the transmit power is $P_{\mathrm{t}_{0}}^{*}$, whereas it gains the connectivity at a much lower number of nodes (e.g., around $N=160$ ) in the case where the transmit power is $2 \times P_{\mathrm{t}_{0}}^{*}$. Hence, transmitting at a power higher than the minimum required level can increase the connectivity range, in the sense that communications can be supported also in sparser networks. In other words:

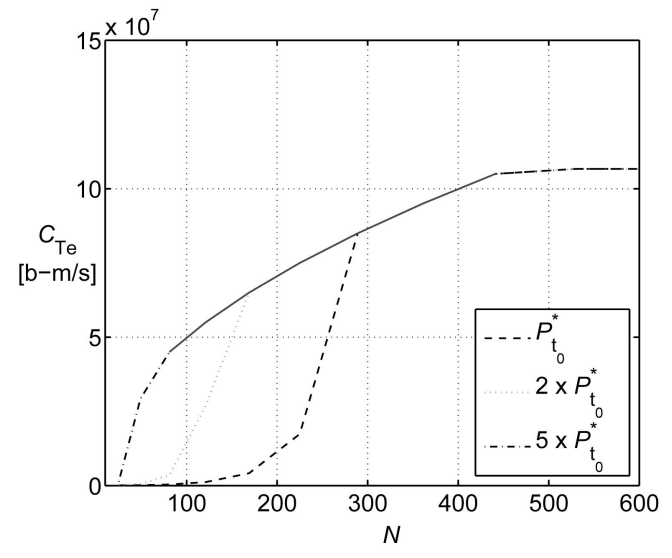

Fig. 14. Effective transport capacity in a network with grid topology.

- if the network is already connected, increasing the transmit power does not increase the effective transport capacity;

- if the network is not connected, increasing the transmit power might make it connected, increasing dramatically the effective transport capacity.

\subsection{Practical Determination of the Optimal Transmit Power}

In this paper, we have presented an analytical approach to determine the optimal common transmit power for network connectivity. Next, we briefly discuss how this optimal transmit power can be determined practically. There are at least two possible strategies based on the capability of the devices. First, in a wireless network where a device can operate with only a single power level, the optimal transmit power can be determined at the design phase. For example, one can estimate the number of nodes and the size of the coverage area that a wireless sensor network will be deployed across and then configure a device such that it operates at the optimal transmit power. Alternatively, if the transmit power of a device is preconfigured at the manufacturing stage, in the network planning phase, one can determine the minimum node spatial density required for this transmit power. This enables a network designer to determine the minimum number of nodes to be deployed in the coverage area.

Second, if each device has an option of changing its transmit power, a distributed power control algorithm can be used. By adapting the COMPOW algorithm [1], the minimum common transmit power for which the route BER satisfies the desired QoS can be determined. Assuming that each device has several discrete power levels, this can be done as follows: Starting from the maximum power level, each node creates a routing table corresponding to this power level. This allows each node to identify all the possible destinations that it could reach. For each destination, a node sends a probing packet to check the route BER at the destination. The same process is repeated for the other available power levels. The minimum power which yields the same routing table as if the maximum power were used and satisfies the required route BER is the optimal power level. 
A careful reader may note that our approach does not take an opportunistic longer hop routing approach, although it may become available when the transmit power becomes larger. However, we emphasize that, although the transmit power is enough to reach a node further than the nearest neighbor, it may still not be enough to reach the destination with the desired level of BER. Certainly, there are trade-offs between choosing the nearest neighbor and choosing the furthest node in a transmission range. The latter is similar to selecting a route with the lowest number of hops to the destination. For more detail on the trade-offs between these two routing strategies, we refer the interested reader to [30], [31].

\section{Related Work}

There are a number of distributed power control algorithms reported in the literature, and it is important to emphasize that they are designed to achieve different objectives. In [32], Foschini and Miljanic proposed a distributed power control algorithm for a cellular network such that the minimum signal-to-interference ratio requirement per user is met. An algorithm proposed by Bambos and Kandukuri [33] attempted to balance the power and delay trade-off by using local information such as last transmitted power level, last interference level, and backlog at the transmitter to determine the appropriate power to transmit in the next time slot. In [34], Monk et al. proposed an algorithm which aims at improving channel efficiency. In [35], Elbatt et al. proposed a power management scheme for improving the end-to-end network throughput. In [25], Chang and Tassiulas proposed a routing algorithm which aims at balancing the energy consumption among the nodes. However, none of these distributed power control algorithms were designed with the objective of ensuring network connectivity.

For our purpose, a distributed power control algorithm should adjust the power such that the minimum power is used to achieve network connectivity. Distributed power control algorithms that are designed with network connectivity and network longevity as their objectives also exist. Some examples of these algorithms are [3], [6], and [27]. One example of distributed power control algorithms, called COMPOW, for determining the minimum common transmit power that keeps the network connected is proposed in [1]. In this algorithm, it is assumed that each device has several discrete power levels to use. The COMPOW algorithm works as follows: Starting from the maximum transmit power level, each node creates a routing table corresponding to this power level. Thus, each node can identify all possible destinations in the network that it could reach. The same process is repeated for every available power level. The common power level to use is the lowest power level that enables each node to reach every destination as if the maximum power were used. By adapting the COMPOW algorithm, the minimum common transmit power for which the route BER satisfies the desired QoS can be determined. However, to the best of our knowledge, none of the previous studies has considered the network connectivity from a communication-theoretic viewpoint [16], [36], where the desired QoS in terms of BER at the end of a multihop route is enforced, as presented in this paper.

A similar analysis of BER at the end of a multihop route, as presented in this paper, also exists in the literature. In [21], Boyer et al. provide a detailed analysis of the route BER in both the decoded relaying multihop channel case and the amplified relaying multihop channel case. In our analysis, we only consider the decoded relaying channel case-that is, we assume that each intermediate node decodes a received bit before forwarding to the next node in the route. However, our BER analysis also includes multiple access interference, whereas this is not considered in [21].

\section{Conclusions}

In this paper, we have investigated the optimal common transmit power for wireless sensor networks. In particular, the optimal common transmit power has been defined as the minimum transmit power sufficient to preserve network connectivity. As opposed to following the conventional graph-theoretic approach, we consider connectivity from a more realistic viewpoint by taking into account the characteristics of a wireless communication channel. Our study shows that, for a given data rate and a given maximum tolerable BER at the end of a multihop route, there exists an optimal transmit power. Moreover, for a given value of the maximum tolerable route BER, there exists a global optimal data rate for which the optimal common transmit power is the minimum possible. This suggests that the data rate, if chosen carefully, can guarantee significant savings in terms of transmit power, prolonging the battery life of devices and network lifetime. This is useful in designing wireless sensor networks. Since the node spatial density of a sensor network is typically known a priori (i.e., the number of sensors and the coverage area are known), a network designer can determine the optimal power and the optimal data rate to operate. Conversely, in the case where the transmit power and the data rate of a sensor node is preconfigured, a network designer can use the approach presented in this paper to determine the minimum node spatial density required to keep the network connected.

However, caution should be exercised when using the common power control scheme proposed in this paper, as its performance is significantly affected by the variation in propagation pathloss. In other words, the common power control scheme performs well in the case where each link in the network experiences similar propagation pathloss. When variation in propagation pathloss occurs, the transmit power needs to be increased substantially even though there is only one link per route which experiences high pathloss. A possible solution to this problem is to add intelligence into the routing protocol. More precisely, a routing protocol that takes link quality as one of its route selection criteria could be an attractive option. However, if it is not possible to avoid a high propagation loss link by means of routing, local power control may be required.

In addition, there are trade-offs between robustness to change in network connectivity and network longevity. Considering a transmit power higher than the optimal value helps preserve connectivity of networks in the presence of node spatial density change. However, this 
comes at the expense of node/network lifetime. Moreover, in a scenario where the network is connected, our results show that increasing the transmit power does not increase the effective transport capacity at all.

Finally, the analytical framework presented in this paper can be further extended to study other interesting scenarios. For example, one may extend this work to estimate the optimal transmit power for other MAC and routing protocols. The channel model can also be modified to take into account multipath fading.

\section{ACKNOWLEDGMENTS}

The authors are grateful to the three anonymous reviewers of this paper and the associate editor, Professor P.R. Kumar of the University of Illinois at Urbana Champaign, whose constructive comments and suggestions helped to improve the manuscript substantially. This work was supported in part by a seed grant from Cylab of Carnegie Mellon University.

\section{REFERENCES}

[1] S. Narayanaswamy, V. Kawadia, R.S. Sreenivas, and P.R. Kumar, "Power Control in Ad-Hoc Networks: Theory, Architecture, Algorithm and Implementation of the COMPOW Protocol," Proc. European Wireless 2002 Next Generation Wireless Networks: Technologies, Protocols, Services, and Applications, pp. 156-162, Feb. 2002.

[2] S. Agarwal, R. Katz, S.V. Krishnamurthy, and S.K. Dao, "Distributed Power Control in Ad-Hoc Wireless Networks," Proc. IEEE Int'l Symp. Personal, Indoor, and Mobile Radio Comm. (PIMRC), vol. 2, pp. F59-F66, Sept. 2001.

[3] R. Ramanathan and R. Rosales-Hain, "Topology Control of Multihop Wireless Networks Using Transmit Power Adjustment," Proc. IEEE Conf. Computer Comm. (INFOCOM), vol. 2, pp. 404-413, Mar. 2000.

[4] T. Elbatt and A. Ephremides, "Joint Scheduling and Power Control for Wireless Ad Hoc Networks," IEEE Trans. Wireless Comm., vol. 3, no. 1, pp. 74-85, Jan. 2004.

[5] R.L. Cruz and A.V. Santhanam, "Optimal Routing, Link Scheduling and Power Control in Multi-Hop Wireless Networks," Proc. IEEE Conf. Computer Comm. (INFOCOM), vol. 1, pp. 702-711, Apr. 2003.

[6] V. Rodoplu and T. Meng, "Minimum Energy Mobile Wireless Networks," IEEE J. Selected Areas in Comm., vol. 17, no. 8, pp. 13331344, Aug. 1999.

[7] C. Bettstetter and J. Zangl, "How to Achieve a Connected Ad Hoc Network with Homogeneous Range Assignment: An Analytical Study with Consideration of Border Effects," Proc. IEEE Int'l Workshop Mobile and Wireless Comm. Network, pp. 125-129, Sept. 2002.

[8] C.-C. Tseng and K.-C. Chen, "Power Efficient Topology Control in Wireless Ad Hoc Networks," Proc. IEEE Wireless Comm. and Networking Conf. (WCNC), vol. 1, pp. 610-615, Mar. 2004.

[9] Q. Dai and J. Wu, "Computation of Minimal Uniform Transmission Power in Ad Hoc Wireless Networks," Proc. IEEE Int'l Conf. Distributed Computing Systems Workshops (ICDCS), pp. 680-684, May 2003.

[10] S. Panichpapiboon, G. Ferrari, and O.K. Tonguz, "Optimal Common Transmit Power in Ad Hoc Wireless Networks," Proc. IEEE Int'l Performance, Computing, and Comm. Conf. (IPCCC), pp. 593-597, Apr. 2005.

[11] I. Akyildiz, W. Su, Y. Sankarasubramaniam, and E. Cayirci, "A Survey on Sensor Networks," IEEE Comm. Magazine, vol. 40, no. 8, pp. 102-114, Aug. 2002.

[12] B. Schrick and M.J. Riezenman, "Wireless Broadband in a Box," IEEE Spectrum, vol. 39, no. 6, pp. 38-43, June 2002.

[13] G. Ferrari, S. Panichpapiboon, N. Wisitpongphan, R. Chokshi, and O.K. Tonguz, "Impact of Mobility on the BER Performance of Multi-Hop Ad Hoc Wireless Networks," Proc. IEEE Global Telecomm. Conf. (GLOBECOM), pp. 882-886, Nov. 2004.

[14] C.E. Perkins, Ad Hoc Networking. Addison-Wesley, 2001.
[15] S. Panichpapiboon, G. Ferrari, and O.K. Tonguz, "Sensor Networks with Random versus Uniform Topology: MAC and Interference Considerations," Proc. IEEE Vehicular Technology Conf. (VTC), pp. 2111-2115, May 2004.

[16] O.K. Tonguz and G. Ferrari, Ad Hoc Wireless Networks: A Communication-Theoretic Perspective. Wiley, 2006.

[17] G. Ferrari and O.K. Tonguz, "Performance of Ad Hoc Wireless Networks with Aloha and PR-CSMA MAC Protocols," Proc. IEEE Global Telecomm. Conf. (GLOBECOM), pp. 2824-2829, Dec. 2003.

[18] N. Abramson, "The Throughput of Packet Broadcasting Channels," IEEE Trans. Comm., vol. COM-25, no. 1, pp. 117-128, Jan. 1977.

[19] O.K. Tonguz and G. Ferrari, "Is the Number of Neighbors in Ad Hoc Wireless Networks a Good Indicator of Connectivity?" Proc. Int'l Zurich Seminar Comm. (IZS '04), pp. 40-43, Feb. 2004.

[20] T.S. Rappaport, Wireless Communications Principles and Practice. Upper Saddle River, N.J.: Prentice-Hall, 1996.

[21] J. Boyer, D. Falconer, and H. Yanikomeroglu, "Multihop Diversity in Wireless Relaying Channels," IEEE Trans. Comm., vol. 52, no. 10, pp. 1820-1830, Oct. 2004.

[22] J. Boyer, D.D. Falconer, and H. Yanikomeroglu, "On the Aggregate SNR of Amplified Relaying Channel," Proc. IEEE Global Telecomm. Conf. (GLOBECOM), vol. 5, pp. 3394-3398, Nov. 2004.

[23] G. Ferrari and O.K. Tonguz, "MAC Protocols and Transport Capacity in Ad Hoc Wireless Networks: Aloha versus PR-CSMA," Proc. IEEE Military Comm. Conf. (MILCOM), pp. 1311-1318, Oct. 2003.

[24] S. Haykin, Communication Systems, fourth ed. John Wiley \& Sons, 2001.

[25] J. Chang and L. Tassiulas, "Energy Conserving Routing in Wireless Ad-Hoc Networks," Proc. IEEE Conf. Computer Comm. (INFOCOM), vol. 1, pp. 22-31, Mar. 2000.

[26] I. Kang and R. Poovendran, "Maximizing Static Network Lifetime of Wireless Broadcast Ad Hoc Networks," Proc. IEEE Int'l Conf. Comm. (ICC), vol. 3, pp. 2256-2261, May 2003.

[27] R. Wattenhofer, L. Li, P. Bahl, and Y. Wang, "Distributed Topology Control for Power Efficient Operation in Multihop Wireless Ad Hoc Networks," Proc. IEEE Conf. Computer Comm. (INFOCOM), vol. 3, pp. 1388-1397, Apr. 2001.

[28] B. Chen, K. Jamieson, H. Balakrishnan, and R. Morris, "Span: An Energy-Efficient Coordination Algorithm for Topology Maintenance in Ad Hoc Wireless Networks," ACM Wireless Networks, vol. 8, no. 5, pp. 481-494, 2002.

[29] S. Panichpapiboon, G. Ferrari, N. Wisitpongphan, and O.K. Tonguz, "Route Reservation in Ad Hoc Networks: Is It a Good Idea?" Proc. IEEE Wireless Comm. and Networking Conf. (WCNC), pp. 2045-2050, Mar. 2005.

[30] N. Wisitpongphan, G. Ferrari, S. Panichpapiboon, J. Parikh, and O.K. Tonguz, "QoS Provisioning Using BER-Based Routing for Ad Hoc Wireless Networks," Proc. IEEE Vehicular Technology Conf. (VTC), May 2005.

[31] G. Ferrari, S.A. Malvassori, M. Bragalini, and O.K. Tonguz, "Physical Layer-Constrained Routing in Ad-Hoc Wireless Networks: A Modified AODV Protocol with Power Control," Proc. Int'l Workshop Wireless Ad-Hoc Networks (IWWAN '05), May 2005.

[32] G. Foschini and Z. Miljanic, "A Simple Distributed Autonomous Power Control Algorithm and Its Convergence," IEEE Trans. Vehicular Technology, vol. 42, no. 4, pp. 641-646, Nov. 1993.

[33] N. Bambos and S. Kandukuri, "Power-Controlled Multiple Access Schemes for Next-Generation Wireless Packet Networks," IEEE Wireless Comm. Magazine, vol. 9, no. 3, pp. 58-64, June 2002.

[34] J. Monk, V. Bharghavan, and W. Hwu, "A Power Controlled Multiple Access Protocol for Wireless Packet Networks," Proc. IEEE Conf. Computer Comm. (INFOCOM), pp. 219-228, Apr. 2001.

[35] T. Elbatt, S. Krishnamurthy, D. Connors, and S. Dao, "Power Management for Throughput Enhancement in Wireless Ad-Hoc Networks," Proc. IEEE Int'l Conf. Comm. (ICC), vol. 3, pp. 15061513, June 2000.

[36] O.K. Tonguz and G. Ferrari, "A Communication-Theoretic Approach to Ad Hoc Wireless Networking," Proc. 2006 Int'l Workshop Wireless Ad-Hoc Networks (IWWAN '06), Invited Paper, June 2006. 


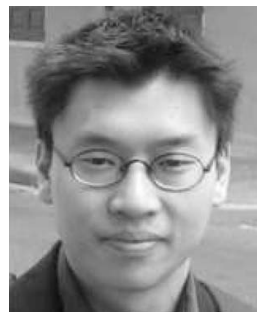

member of the IEEE.

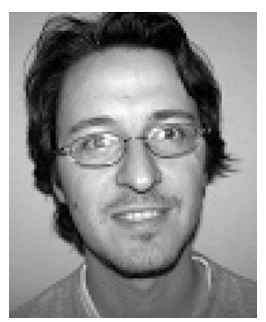

Sooksan Panichpapiboon (S'05) received the BS and MS degrees in electrical and computer engineering from Carnegie Mellon University (CMU), Pittsburgh, Pennsylvania, in 2000 and 2002, respectively. He is currently a $\mathrm{PhD}$ candidate in the Electrical and Computer Engineering (ECE) Department at CMU. His research interests include ad hoc wireless networks, sensor networks, medium access control, and performance modeling. He is a student

Gianluigi Ferrari (S'97-M'03) received the "Laurea" degree (5-year program) (summa cum laude) and the PhD degree in electrical engineering from the University of Parma, Italy, in October 1998 and January 2002, respectively. From July 2000 to December 2001, he was a visiting scholar at the Communication Sciences Institute, University of Southern California, Los Angeles. Since 2002, he has been a research professor in the Department of Information Engineering, University of Parma. Between 2002 and 2004, he visited the Electrical and Computer Engineering Department at Carnegie Mellon University, Pittsburgh, Pennsylvania, several times as a research associate. He has published more than 70 papers in leading international conferences and journals. He is a coauthor of the books Detection Algorithms for Wireless Communications, with Applications to Wired and Storage Systems (John Wiley \& Sons, 2004), Teoria della Probabilitá e Variabili Aleatorie con Applicazioni (McGraw-Hill, 2005), and Ad Hoc Wireless Networks: A Communication-Theoretic Perspective (John Wiley \& Sons, 2006). He is listed in Marquis' Who's Who in the World and Who's Who in Science and Engineering, Madison's Who's Who in the World, and in 2000 Outstanding Intellectuals of the 21st Century (International Biographical Centre, UK, 2005). His research interests include digital communication systems design, adaptive signal processing (with particular emphasis on iterative detection techniques for channels with memory), information theory, and ad hoc wireless networking. He was a corecipient of a Best Student Paper Award at the 2006 International Workshop on Wireless Ad-Hoc Networks (IWWAN '06). He acts as a frequent reviewer for many international journals and conferences. He also acts as a technical program member for several international conferences (IEEE ICC, IEEE WirelessCom, IEEE IWCMC, Accessnets, IEEE WCNC, IWWAN, and IASTED CSA). $\mathrm{He}$ is a member of the IEEE.

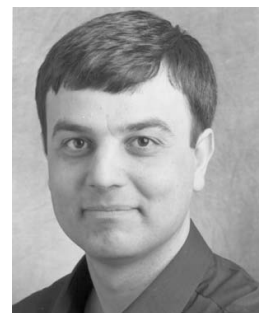

Ozan K. Tonguz received the BSc degree from the University of Essex, England, in 1980, and the MSc and PhD degrees from Rutgers University, New Brunswick, New Jersey, in 1986 and 1990, respectively, all in electrical engineering. He currently serves as a tenured full professor in the Department of Electrical and Computer Engineering at Carnegie Mellon University (CMU). Before joining $\mathrm{CMU}$ in August 2000, he was with the Electrical and Computer Engineering Department at the State University of New York at Buffalo (SUNY/Buffalo). He joined SUNY/Buffalo in 1990 as an assistant professor, where he was granted early tenure and promoted to associate professor in 1995, and to full professor in 1998. Prior to joining academia, he was with Bell Communications Research (Bellcore) between 1988-1990 doing research in optical networks and communication systems. His current research interests are in wireless networks and communication systems, high-speed networking, optical networks, satellite communications, bioinformatics, and security. He has published more than 200 technical papers in IEEE journals and conference proceedings. He is well-known for his contributions in optical networks (optical transmission-access-networking, especially radio over fiber networks, coherent lightwave transmission systems, and amplified direct-detection lightwave transmission sytems) and his recent work on iCAR (the Integrated Cellular and Ad Hoc Relay Systems) is internationally acclaimed as well. He is the author of the book Ad Hoc Wireless Networks: A Communication-Theoretic Perspective (Wiley and Sons, 2006) and was also the architect of the "High Performance Waveform (HPW)" that was implemented in Harris RF Communications' AN/PRC-117f UHF band man-pack tactical radio. His industrial experience includes periods with Bell Communications Research, CTI Inc., Harris RF Communications, Aria Wireless Systems, Clearwire Technologies, Nokia Networks, Nokia Research Center, Neuro Kinetics, Asea Brown Boveri (ABB), General Motors (GM), and Intel. He currently serves as a consultant for several companies, law firms, and government agencies in the US and Europe in the broad area of telecommunications and networking. $\mathrm{He}$ is also a codirector (thrust leader) of the Center for Wireless and Broadband Networking Research at Carnegie Mellon University. More details about his research interests, research group, projects, and publications can be found at http:// www.ece.cmu.edu/ tonguz/. In addition to serving on the technical program committees of several IEEE conferences (such as INFOCOM, GLOBECOM, ICC, VTC, and WCNC) and symposia in the areas of wireless communications and optical networks, Dr. Tonguz currently serves or has served as an associate editor for the IEEE Transactions on Communications, IEEE Communications Magazine, and IEEE Journal of Lightwave Technology. He was a guest editor of the special issue of the IEEE Journal of Lightwave Technology and IEEE Journal on Selected Areas in Communications on multiwavelength optical networks and technology, published in 1996, and a guest editor of the special issue of the Journal of Mobile Multimedia on advanced mobile technologies for health care applications, published in 2006. He is a member of the IEEE.

$\triangleright$ For more information on this or any other computing topic, please visit our Digital Library at www.computer.org/publications/dlib. 